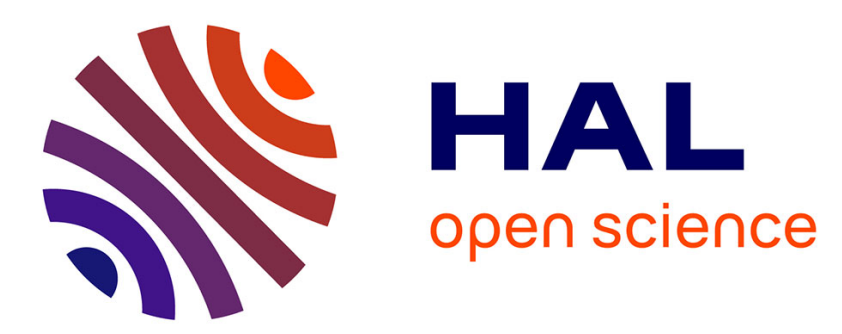

\title{
Operator splitting methods with error estimator and adaptive time-stepping. Application to the simulation of combustion phenomena
}

Stéphane Descombes, Max Duarte, Marc Massot

\section{- To cite this version:}

Stéphane Descombes, Max Duarte, Marc Massot. Operator splitting methods with error estimator and adaptive time-stepping. Application to the simulation of combustion phenomena. Roland Glowinski; Stanley Osher; Wotao Yin. Splitting Methods in Communication, Imaging, Science, and Engineering, Springer International Publishing, pp.1-13, 2015, 78-3-319-41589-5. 10.1007/978-3-319-41589-5 . hal$01183745 \mathrm{v} 2$

\section{HAL Id: hal-01183745 \\ https://hal.science/hal-01183745v2}

Submitted on 17 Nov 2015

HAL is a multi-disciplinary open access archive for the deposit and dissemination of scientific research documents, whether they are published or not. The documents may come from teaching and research institutions in France or abroad, or from public or private research centers.
L'archive ouverte pluridisciplinaire HAL, est destinée au dépôt et à la diffusion de documents scientifiques de niveau recherche, publiés ou non, émanant des établissements d'enseignement et de recherche français ou étrangers, des laboratoires publics ou privés. 


\title{
Operator splitting methods with error estimator and adaptive time-stepping. Application to the simulation of combustion phenomena
}

\author{
Stéphane Descombes* $\quad$ Max Duarte ${ }^{\dagger} \quad$ Marc Massot $^{\ddagger}$
}

November 17, 2015

\begin{abstract}
Operator splitting techniques were originally introduced with the main objective of saving computational costs. A multi-physics problem is thus split in subproblems of different nature with a significant reduction of the algorithmic complexity and computational requirements of the numerical solvers. Nevertheless, splitting errors are introduced in the numerical approximations due to the separate evolution of the split subproblems and can compromise a reliable reproduction of the coupled dynamics. In this chapter we present a numerical technique to estimate such splitting errors on the fly and dynamically adapt the splitting time steps according to a user-defined accuracy tolerance. The method applies to the numerical solution of time-dependent stiff PDEs, illustrated here by propagating laminar flames investigated in combustion applications.
\end{abstract}

\section{Context and motivation}

Let us consider a scalar reaction-diffusion equation

$$
\left.\begin{array}{ll}
\partial_{t} u-\partial_{x}^{2} u=f(u), & x \in \mathbb{R}, t>0, \\
u(x, 0)=u_{0}(x), & x \in \mathbb{R},
\end{array}\right\}
$$

and represent the solution $u(., t)$ as $T^{t} u_{0}$, where $T^{t}$ is the semi-flow associated with (1). Given $v_{0}$ and $w_{0}$, an operator splitting approach amounts to consider the following subproblems:

$$
\left.\begin{array}{ll}
\partial_{t} v-\partial_{x}^{2} v=0, & x \in \mathbb{R}, t>0, \\
v(x, 0)=v_{0}(x), & x \in \mathbb{R},
\end{array}\right\}
$$

and

$$
\left.\begin{array}{ll}
\partial_{t} w=f(w), & x \in \mathbb{R}, t>0, \\
w(x, 0)=w_{0}(x), & x \in \mathbb{R} .
\end{array}\right\}
$$

*Université Nice Sophia Antipolis, CNRS, LJAD, UMR 7351, 06100 Nice, France and INRIA Sophia Antipolis-Mediterrané Research Center, Nachos project-team, 06902 Sophia Antipolis Cedex, France (stephane.descombes@unice.fr).

${ }^{\dagger}$ CCSE, Lawrence Berkeley National Laboratory, 1 Cyclotron Rd. MS 50A-1148, Berkeley, CA 94720, USA and CD-adapco, 200 Shepherds Bush Road, London W6 7NL, UK (max.duarte@cd-adapco.com).

${ }^{\ddagger}$ CNRS UPR 288, Laboratoire EM2C, CentraleSupélec, Fédération de Mathématiques de l'École Centrale Paris, CNRS FR 3487, Grande Voie des Vignes, 92295 Chatenay-Malabry Cedex, France (marc.massot@centralesupelec.fr). 
We denote by $X^{t} v_{0}$ and $Y^{t} w_{0}$, respectively, the solutions of (2) and (3). The Lie (or Lie-Trotter [54]) splitting approximations to the solution of problem (1) are thus given by

$$
\mathcal{L}_{1}^{t} u_{0}=X^{t} Y^{t} u_{0}, \quad \mathcal{L}_{2}^{t} u_{0}=Y^{t} X^{t} u_{0} .
$$

Lie approximations are of first order in time; second order can be achieved by using symmetric Strang (or Marchuk [40]) formulas [52] to obtain

$$
\mathcal{S}_{1}^{t} u_{0}=X^{t / 2} Y^{t} X^{t / 2} u_{0}, \quad \mathcal{S}_{2}^{t} u_{0}=Y^{t / 2} X^{t} Y^{t / 2} u_{0} .
$$

Even though higher order splitting schemes have been also developed, more sophisticated numerical implementations are required and their applicability is currently limited to specific linear or nonstiff problems (see, e.g., [17, 11, 33, 9] and discussions therein). The main advantage of such a splitting approach is that problems of different mathematical nature, in this case diffusion and reaction equations, can be solved separately with dedicated numerical methods. The latter involves a significant reduction of the algorithmic complexity of the overall method to advance the fully coupled problem with a potential reduction of computational requirements.

However, the separate time evolution of the split subproblems during a given splitting time step $\Delta t$ introduces the so-called splitting errors. These errors have been mathematically characterized in the literature for general nonlinear problems and sufficiently small splitting time steps, relying mainly on the Baker-Campbell-Hausdorff formula on composition of exponentials together with Lie derivative calculus (see, e.g., [31] for ODEs and [35] for PDEs). In particular, Lanser \& Verwer explicitly derived in [39] the splitting errors arising in the solution of reaction-diffusion-convection equations. Within this theoretical framework and considering an appropriate functional space, the following estimates can be thus derived for the scalar nonlinear reaction-diffusion equation (1).

Theorem 1. Given $\mathrm{C}_{\mathrm{b}}^{\infty}(\mathbb{R})$, the space of functions of class $\mathrm{C}^{\infty}$ on $\mathbb{R}$ and bounded over $\mathbb{R}$, let us introduce the Schwartz space $\mathbb{S}(\mathbb{R})$ defined by

$$
\mathbb{S}(\mathbb{R})=\left\{g \in \mathrm{C}^{\infty}(\mathbb{R})\left|\sup _{v \in \mathbb{R}}\right| v^{\alpha_{1}} \partial_{v}^{\alpha_{2}} g(v) \mid<\infty \quad \text { for all integers } \alpha_{1}, \alpha_{2}\right\}
$$

and define the space $\mathbb{S}_{1}(\mathbb{R})$ made out of functions $v$ belonging to $\mathrm{C}_{\mathrm{b}}^{\infty}(\mathbb{R})$ such that $v^{\prime}$ belongs to $\mathbb{S}(\mathbb{R})$.

Assume that $u_{0}$ belongs to $\mathbb{S}_{1}(\mathbb{R})$ and that $f$ belongs to $\mathrm{C}^{\infty}(\mathbb{R})$. For $\Delta t$ small enough, the following asymptotics hold

$$
T^{\Delta t} u_{0}-\mathcal{L}_{2}^{\Delta t} u_{0}=\frac{\Delta t^{2}}{2} f^{\prime \prime}\left(u_{0}\right)\left(\partial_{x} u_{0}\right)^{2}+\mathcal{O}\left(\Delta t^{3}\right)
$$

and

$$
\begin{aligned}
T^{\Delta t} u_{0}-\mathcal{S}_{2}^{\Delta t} u_{0}= & \frac{\Delta t^{3}}{24}\left[f^{\prime}\left(u_{0}\right) f^{\prime \prime}\left(u_{0}\right)+f\left(u_{0}\right) f^{(3)}\left(u_{0}\right)\right]\left(\partial_{x} u_{0}\right)^{2} \\
& -\frac{\Delta t^{3}}{12} f^{(4)}\left(u_{0}\right)\left(\partial_{x} u_{0}\right)^{4}-\frac{\Delta t^{3}}{3} f^{(3)}\left(u_{0}\right)\left(\partial_{x} u_{0}\right)^{2} \partial_{x}^{2} u_{0} \\
& -\frac{\Delta t^{3}}{6} f^{\prime \prime}\left(u_{0}\right)\left(\partial_{x}^{2} u_{0}\right)^{2}+\mathcal{O}\left(\Delta t^{4}\right) .
\end{aligned}
$$

Proof. It suffices to consider the Baker-Campbell-Hausdorff formula (see [31, 35]) and compute the corresponding Lie brackets (commutators in the case of linear operators) containing the Lie derivatives associated with the nonlinear function $f$ and the Laplace operator $\Delta$ (see [19]).

Similar estimates can be derived for $\mathcal{L}_{1}^{\Delta t} u_{0}$ and $\mathcal{S}_{1}^{\Delta t} u_{0}$. More refined estimates that characterize the dependences with respect to the norms of the initial data and the nonlinearity can be also obtained using exact error representations. The following theorem shows, for instance, the representation of $T^{\Delta t} u_{0}-\mathcal{L}_{2}^{\Delta t} u_{0}$. 
Theorem 2. Let us denote by $D_{2}$ the derivative with respect to the initial condition, under the same assumptions of Theorem 1 we have

$$
\begin{aligned}
T^{\Delta t} u_{0}-\mathcal{L}_{2}^{\Delta t} u_{0}= & \int_{0}^{\Delta t} \int_{0}^{s} D_{2} T^{t-s}\left(Y^{s} X^{s} u_{0}\right) \exp \left(\int_{0}^{s-r} f^{\prime}\left(Y^{\sigma+r} X^{s} u_{0}\right) d \sigma\right) \times \\
& f^{\prime \prime}\left(Y^{r} X^{s} u_{0}\right) \exp \left(2 \int_{0}^{r} f^{\prime}\left(Y^{\sigma} X^{s} u_{0}\right) d \sigma\right)\left(\partial_{x} X^{s} u_{0}\right)^{2} d r d s .
\end{aligned}
$$

Similar representations can be derived for $\mathcal{L}_{1}^{\Delta t} u_{0}, \mathcal{S}_{1}^{\Delta t} u_{0}$, and $\mathcal{S}_{2}^{\Delta t} u_{0}$. These results are due to a long series of papers and especially those of Michelle Schatzman, a great contributor to operator splitting methods. Originally introduced in [48] for linear operators, these exact representations of the local errors have been extended in a more general functional setting in $[24,22]$ and in the nonlinear case in $[23,21]$.

Even though theoretical estimates of splitting errors can be formally established for general problems like (1), computing them in practice in multi-dimensional configurations or for more complex models may rapidly become cumbersome. Developing a splitting error estimator based on these theoretical estimates can hence be inappropriate except for particular configurations like, for example, linear problems as proposed in [2]. On the other hand, splitting solvers that do not account for splitting errors may yield numerical approximations that poorly reproduce the coupled physical dynamics of the problem under investigation. The latter is even more relevant if one takes into account that practical considerations often suggest the use of relatively large splitting time steps in order to ease heavy computational costs related to the numerical simulation of complex applications. In what follows we present a numerical strategy to estimate splitting errors on the fly and hence adapt splitting time steps to guarantee numerical approximations within a user-defined accuracy tolerance. The scheme was originally introduced in [19] along with its corresponding mathematical analysis. Throughout this chapter we consider the scalar nonlinear reaction-diffusion equation (1), although the same ideas are easily extended to multi-dimensional or more complex configurations, as well as to other time-dependent stiff PDEs.

\section{Splitting error estimator and adaptive time-stepping}

In general an adaptive time-stepping technique relies on a dynamic numerical estimate of local errors; time steps are consequently set according to a pre-defined accuracy tolerance. In our case estimating the splitting errors, for instance, (6) and (7), constitutes the key issue since the physics of the problem may be substantially altered by the splitting procedure. Inspired by ODE solvers one way to compute such an estimate considers a lower order scheme, embedded if possible in the main numerical integration solver (see, e.g., [32]). This is a standard approach, for instance, for Runge-Kutta methods.

Based on the $\mathcal{S}_{2}$-scheme in (5), let us consider the shifted Strang formula introduced in [19],

$$
\mathcal{S}_{2, \varepsilon}^{\Delta t} u_{0}=Y^{(1 / 2-\varepsilon) \Delta t} X^{\Delta t} Y^{(1 / 2+\varepsilon) \Delta t} u_{0},
$$

for $\varepsilon$ in $(-1 / 2,0) \cup(0,1 / 2)$. To simplify the notations, we will denote $\mathcal{S}_{2}^{t}$ by $\mathcal{S}^{t}$ and $\mathcal{S}_{2, \varepsilon}^{t}$ by $\mathcal{S}_{\varepsilon}^{t}$. Similar to Theorem 1 the following one was demonstrated in [19].

Theorem 3. Assume that $u_{0}$ belongs to $\mathbb{S}_{1}(\mathbb{R})$ and that $f$ belongs to $\mathrm{C}^{\infty}(\mathbb{R})$. For $\Delta t$ and $\varepsilon$ small 
enough, the following asymptotic holds

$$
\begin{aligned}
T^{\Delta t} u_{0}-\mathcal{S}_{\varepsilon}^{\Delta t} u_{0}= & -\varepsilon \Delta t^{2} f^{\prime \prime}\left(u_{0}\right)\left(\partial_{x} u_{0}\right)^{2} \\
& \frac{\Delta t^{3}}{24}\left[f^{\prime}\left(u_{0}\right) f^{\prime \prime}\left(u_{0}\right)+f\left(u_{0}\right) f^{(3)}\left(u_{0}\right)\right]\left(\partial_{x} u_{0}\right)^{2} \\
& -\frac{\Delta t^{3}}{12} f^{(4)}\left(u_{0}\right)\left(\partial_{x} u_{0}\right)^{4}-\frac{\Delta t^{3}}{3} f^{(3)}\left(u_{0}\right)\left(\partial_{x} u_{0}\right)^{2} \partial_{x}^{2} u_{0} \\
& -\frac{\Delta t^{3}}{6} f^{\prime \prime}\left(u_{0}\right)\left(\partial_{x}^{2} u_{0}\right)^{2}+\mathcal{O}\left(\varepsilon \Delta t^{3}\right)+\mathcal{O}\left(\Delta t^{4}\right) .
\end{aligned}
$$

Proof. See [19] Theorem 3.2.

Therefore, just like Lie schemes, the shifted Strang formula (8) yields first order approximations. For $\varepsilon$ equal to $-1 / 2$ (resp., 0) estimate (9) becomes (6) (resp., (7)). In particular, from (7) and (9), we have that

$$
\mathcal{S}^{\Delta t} u_{0}-\mathcal{S}_{\varepsilon}^{\Delta t} u_{0}=\varepsilon \Delta t^{2} f^{\prime \prime}\left(u_{0}\right)\left(\partial_{x} u_{0}\right)^{2}+\mathcal{O}\left(\varepsilon \Delta t^{3}\right) .
$$

Given $u_{0}$, we can thus compute two splitting approximations,

$$
\left(\begin{array}{c}
\mathcal{S}^{\Delta t} u_{0} \\
\mathcal{S}_{\varepsilon}^{\Delta t} u_{0}
\end{array}\right)=\left(\begin{array}{c}
Y^{\Delta t / 2} X^{\Delta t} Y^{\Delta t / 2} u_{0} \\
Y^{(1 / 2-\varepsilon) \Delta t} X^{\Delta t} Y^{(1 / 2+\varepsilon) \Delta t} u_{0}
\end{array}\right)
$$

where the $\mathcal{S}_{\varepsilon}$-scheme is a lower order, embedded method with respect to the standard $\mathcal{S}$-scheme. Embedding is accomplished as long as $\varepsilon$ is different from $-1 / 2$, i.e., $\mathcal{S}_{2, \varepsilon}^{t}$ is not $\mathcal{L}_{2}^{t}$. Taking into account that

$$
\begin{aligned}
\mathcal{S}^{\Delta t} u_{0}-\mathcal{S}_{\varepsilon}^{\Delta t} u_{0} & =\mathcal{S}^{\Delta t} u_{0}-T^{\Delta t} u_{0}+T^{\Delta t} u_{0}-\mathcal{S}_{\varepsilon}^{\Delta t} u_{0} \\
& =\mathcal{O}\left(\Delta t^{3}\right)+\mathcal{O}\left(\Delta t^{2}\right) \approx \mathcal{O}\left(\Delta t^{2}\right)
\end{aligned}
$$

we define a splitting error estimator, err, and for a given accuracy tolerance, $\eta$, the following must be verified

$$
\operatorname{err}=\left\|\mathcal{S}^{\Delta t} u_{0}-\mathcal{S}_{\varepsilon}^{\Delta t} u_{0}\right\| \leq \eta,
$$

to assure local splitting errors bounded by $\eta$. Supposing that $\operatorname{err} \approx C \Delta t^{2}$ following (11), we define a new splitting time step, $\Delta t_{\text {new }}$, such that $\eta \approx C \Delta t_{\text {new }}^{2}$. Therefore, the adaptive time-stepping is defined by

$$
\Delta t_{\text {new }}=v \Delta t \sqrt{\frac{\eta}{e r r}}
$$

with a security factor $v>0$, close to 1 .

To summarize, given the numerical approximation $u_{n}$ at a given time and the splitting time step $\Delta t_{n}$, the time-stepping technique for a given $\eta$ is performed as follows:

1. Compute both splitting approximations: $\mathcal{S}^{\Delta t_{n}} u_{n}$ and $\mathcal{S}_{\varepsilon}^{\Delta t_{n}} u_{n}$, following (10).

2. Compute the splitting error estimator: $\mathrm{err}=\left\|\mathcal{S}^{\Delta t_{n}} u_{n}-\mathcal{S}_{\varepsilon}^{\Delta t_{n}} u_{n}\right\|$, and the new splitting time step, $\Delta t_{\text {new }}$, with (12).

3. If err $\leq \eta$, the solution $\mathcal{S}^{\Delta t_{n}} u_{n}$ is accepted: $u_{n+1}=\mathcal{S}^{\Delta t_{n}} u_{n}$ and $\Delta t_{n+1}=\Delta t_{\text {new }}$. Otherwise, the solution is rejected and the time-stepping technique is restarted with $\Delta t_{n}=\Delta t_{\text {new }}$.

Notice that whenever a lower order, embedded scheme is used to estimate local errors, the error estimator is actually measuring the numerical errors associated with the low order approximation, computed here by the $\mathcal{S}_{\varepsilon}$-scheme. The splitting error in the numerical solution is therefore overestimated since the $\mathcal{S}$-scheme is formally of higher order. However, this is a safety choice to guarantee numerical approximations within the user-defined accuracy tolerance. In particular a 
complementary numerical procedure was developed in [19] in order to dynamically choose $\varepsilon$ such that the estimator err yields closer estimates to the actual splitting errors $\left\|T^{\Delta t_{n}} u_{n}-\mathcal{S}^{\Delta t_{n}} u_{n}\right\|$, even for relatively large splitting time steps.

The shifted Strang formula (8) could also become the $\mathcal{L}_{1}$-scheme, if we let $\varepsilon$ be equal to $1 / 2$. In this case the $\mathcal{L}_{1}$-scheme in (4) acts as the lower order, embedded method for the $\mathcal{S}_{2}$-scheme, as proposed in [37] for non-stiff problems. Nevertheless, it is well-known in the context of stiff PDEs that order reductions may arise, due to short-life transients associated with the fastest variables, when one considers splitting time steps larger than the fastest scales. In particular it has been mathematically proved in $[51,20,38]$ that better performances are expected when the splitting scheme ends with the stiffest operator. Having the $\mathcal{S}_{\varepsilon}$-scheme as the embedded scheme, built analogously to the standard Strang formula with the same stiffest operator as the ending step, involves similar behaviors in terms of order reduction and overall numerical performance for relatively large splitting time steps. Finally, the mathematical analysis was conducted in [19] in the case of the scalar nonlinear reaction-diffusion equation (1); however, the asymptotic estimate (9) would in general remain of $\mathcal{O}\left(\varepsilon \Delta t^{2}\right)$ when considering other PDEs.

\section{Dedicated splitting solver for stiff PDEs}

An operator splitting approach allows one to use appropriate solvers for each split subproblems. In particular for splitting schemes resulting from composition methods like (4)-(5), the numerical stability of the splitting scheme is assured depending on the stability properties of these solvers. That is, if the numerical solvers used to advance each split subproblems are stable during a given splitting time step, then the splitting approximation will remain stable. The latter involves that relatively large splitting time steps can be considered without having any numerical issue; however, the validity of results may be undermined by the splitting errors. The technique summarized in $\S 2$ aims at tracking these errors, but it relies in practice on a splitting solver that must be capable to cope with stiff PDEs.

Let us make the following observations on splitting schemes for stiff PDEs:

1. The exact solutions of the split subproblems are considered in the classical analysis of splitting schemes, given here by the semi-flows $X^{t}$. and $Y^{t}$, associated with equations (2) and (3), respectively. This is also the case for the $\mathcal{S}_{\varepsilon}$-scheme introduced in [19].

2. For sufficiently large splitting time steps, the intermediate splitting approximations may drift away from the coupled dynamics. The latter may introduce potentially fast transients or boundary layers immediately after a split operator has been applied [57, 45].

3. As previously said, for relatively large splitting time steps, better performances are expected with splitting schemes that end with the stiffest operator [51, 20,38].

We consider here the dedicated splitting solver developed in [28] to address the latter three remarks. This solver was originally conceived for stiff reaction-diffusion models, but the same ideas can be extended to other configurations.

In terms of the numerical methods used to solve the split subproblems, we consider one-step and high order schemes with appropriate stability properties and time-stepping features, based on the following precepts [28]:

- One-step schemes are preferred over multi-step ones because of the initial procedure needed by the latter to start the algorithm. For splitting schemes this starting procedure would be performed at least once at every splitting time step and might coincide with the fast transient regimes, hence involving more computational effort (see, e.g., the study conducted in [55]).

- Integration schemes of approximation order higher than the splitting method are chosen such that the corresponding integration errors remain lower than the splitting ones. In this way 
integration errors do not interfere with the splitting ones and the global accuracy of the method is set by the splitting scheme as considered in the corresponding mathematical analysis.

- Methods with time-stepping features based on stability or a user-defined accuracy tolerance are preferred, computing as many time steps as necessary within a given splitting time step. The latter is particularly relevant to adapt time steps during short non-physical transients that may arise within splitting time steps. The splitting time step can be therefore defined independently of the numerical integration of the split subproblems avoiding stability constraints associated with mesh size or stiff source time scales. When only one integration time step is needed and it is therefore equal to the splitting one, the higher order solver yields numerical errors potentially lower than the splitting ones.

- When implicit methods are used to cope with the stiffness of a given subproblem, $L$-stable schemes should be considered to rapidly damp out potentially fast transients. As before onestep schemes are favored as multi-step methods cannot be $L$-stable with an order higher than $2[14]$.

Further discussions on these aspects can be found in [25].

As an illustration, the splitting technique proposed in [28] to solve stiff reaction-diffusion equations like (1) considers the following solvers: Radau5 [32] for the reaction subproblem (3) and the ROCK4 method [1] for the diffusion one (2). Radau5 is a fifth order implicit Runge-Kutta method exhibiting $A$ - and $L$-stability properties to efficiently solve stiff systems of ODEs. However, the high performance of implicit Runge-Kutta methods for stiff ODEs is adversely affected when applied to large systems of nonlinear equations arising in the numerical integration of semi-discrete stiff PDEs. Significant effort is actually required to achieve numerical implementations that solve the corresponding algebraic problems at reasonable computational expenses. A splitting approach offers a much simpler alternative since the split subproblem (3) becomes a system of ODEs which can be separately solved point-wise over the computational domain. On the other hand, ROCK4 is a fourth order stabilized explicit Runge-Kutta method with extended stability domain along the negative real axis, well suited to numerically treat mildly stiff elliptic operators. The diffusion equation (2) is thus solved over the entire domain with an explicit scheme and therefore with a limited memory requirement with respect to an implicit one. Both methods implement adaptive time-stepping techniques to guarantee computations within a prescribed accuracy tolerance.

Within this framework one can prescribe relatively fine tolerances for the numerical solvers, Radau5 and ROCK4, and the only input parameter of the splitting solver is the splitting time step. In [28] a constant splitting time step was considered to simulate propagating reaction waves. In this case the wavefront velocity is retained as the key physical parameter to define a splitting time step that guarantees a sufficiently accurate resolution of the coupled dynamics. The numerical solvers are thus in charge of solving the split subproblems during the given splitting time step, assuring the numerical stability of the computations and coping with the stiffness of the equations. The performance of this strategy was assessed in the context of multi-dimensional chemical waves [28] and for a model of human ischemic stroke with several variables and complex mechanisms in the stiff source terms $[27,29]$. In both cases the $\mathcal{S}_{2}$-scheme that ends with the reaction operator was used according to the theoretical insights derived in [20] for stiff nonlinear reaction-diffusion equations. Notice that localized reacting fronts are simulated in the case of propagating waves; therefore, intense computation of the stiff reaction problem (3) is required only along the fronts where important reactive activity is present. Consequently, Radau5 adapts its time steps only where it is necessary with important computational enhancements, yielding local time steps that may substantially differ according to the vicinity of the fronts. The latter cannot be done in the same simple way without splitting the original model.

While preliminary studies were required to determine an appropriate constant time step for propagating waves (as shown in $[28,27]$ ), they are no longer necessary if one considers the adaptive splitting technique with error estimator introduced in [19] and previously described in $\S 2$. These 
methods complement each other since the adaptive strategy also requires a dedicated splitting solver to guarantee the theoretical framework of the analysis and to efficiently handle in practice stiffness and stability issues. As a result we end up with an adaptive, dedicated splitting solver for stiff PDEs. The only input parameter now is the splitting tolerance accuracy $\eta$, noting that the accuracy parameters of the numerical solvers must be set lower than $\eta$. Notice that lower order schemes for the split subproblems could be also used with the adaptive splitting technique as long as these solvers implement adaptive time-stepping with error control and their accuracy tolerances are set lower than the splitting one. However, for a given prescribed accuracy, higher order solvers as proposed in [28] yield solutions with potentially larger time steps.

\section{Operator splitting for combustion problems}

Operator splitting methods have been used in the literature for decades, and were widely implemented and exploited for combustion problems to overcome classical restrictions on computational resources (see, e.g., [30, 15, 59, 43, 47, 49, 50, 46, 13, 44]). A good example is given by the numerical strategy developed in $[36,42]$ for reactive flows in a low Mach number formulation with detailed chemical kinetics and transport parameters. The splitting scheme introduced by these authors combines the implicit multi-step VODE solver [10] for stiff ODEs for the chemical reaction terms with a second order explicit RKC scheme [56, 58] for the diffusion problem. In this way important gains of computational efficiency are achieved with a splitting time step not limited by the stiff reactive scales and set according to the extended stability domain of the RKC solver (when convective stability constraints are less restrictive). Moreover, another efficient low Mach solver was introduced in [16] with an operator splitting method coupled with an AMR (Adaptive Mesh Refinement) technique [8,3]. The problem is thus solved level-wise throughout a set of grids with different resolution, with splitting time steps set by the corresponding CFL condition associated with each grid size. Reaction terms are locally solved with VODE. With these bases, further developments in terms of algorithm implementation and parallel computing techniques led to the effective simulation of three-dimensional turbulent premixed flames with detailed chemistry (see, e.g., [5, 4]), a remarkable achievement for laboratory-scale turbulent flames (see, e.g., [7, 6]).

Considering the state of art and these recent advances, one may note as previously remarked that splitting schemes favor the use of dedicated numerical solvers of different nature as well as straightforward coupling with other techniques, with important gains in computational efficiency. Nevertheless, there are some open issues related to the construction of splitting schemes and the interaction of splitting errors with those originating from the inner implicit-explicit solvers (the influence of the latter ones on the global integration error was numerically illustrated, for instance, in $[36,42])$. In particular a critical matter underlined in the literature is the lack of precise criteria to properly choose the splitting time steps according to the physical decoupling capabilities of the problem and for a given accuracy. Another question is the extension of these strategies to highly dynamic problems for which neither a constant nor a stability-based variable splitting time step is adequate, taking into account that the explicit schemes are intended to handle the slow, non-stiff part of the equations.

To address these problems the adaptive operator splitting scheme, briefly recalled in $\S 2$, was considered in [26] combined with a dedicated splitting solver for stiff time dependent PDEs built in practice under the precepts described in §3. Additionally, this splitting solver was coupled in [28] with a dynamic mesh refinement technique based on multiresolution (MR) analysis [34, 12, 41]. For a given semi-discretized problem, the MR mathematical background allows a better monitoring of numerical errors introduced by the compressed spatial representations with respect to the original uniform grid discretization. The resulting time-space adaptive solution scheme was thus described and analyzed in [26] and constitutes a fundamental building block for solvers used in combustion simulations. It provides an efficient algorithm in terms of both memory storage and computational performance, which allows multi-dimensional simulations assuring a given error tolerance, fixed in 
advance by the user.

The study conducted in [26] considered multi-dimensional laminar flames interacting with vortex structures, including the propagation of flame fronts and self-ignition processes of reactive mixtures. In what follows we recall some interesting aspects resulting from the numerical simulation of the ignition process and generation of a diffusion flame, investigated in [26].

\section{Numerical illustration}

Let us consider the mathematical model derived in [53] to investigate the ignition dynamics of a diffusion flame, formed while a reactive layer is being rolled-up in a vortex. The hydrodynamics is decoupled from species and energy transport equations by adopting the standard thermo-diffusive approximation, leading to a reaction-diffusion-convection model. A two-dimensional computational domain is considered where pure and fresh hydrogen at temperature $T_{\mathrm{F}, 0}$ initially occupies the upper half part, while the remaining lower part of the domain is occupied by hot air at $T_{\mathrm{O}, 0}$. By defining a Schvab-Zeldo'vich variable $Z$ and a reduced temperature $\theta$ given by

$$
\theta=\frac{T-T_{\mathrm{O}, 0}}{T_{\mathrm{F}, 0}-T_{\mathrm{O}, 0}},
$$

the mathematical model is given by a system of equations of the form [53]:

$$
\left.\begin{array}{l}
\partial_{t} Z+v_{x} \partial_{x} Z+v_{y} \partial_{y} Z-\left(\partial_{x}^{2} Z+\partial_{y}^{2} Z\right)=0, \\
\partial_{t} \theta+v_{x} \partial_{x} \theta+v_{y} \partial_{y} \theta-\left(\partial_{x}^{2} \theta+\partial_{y}^{2} \theta\right)=F(Z, \theta),
\end{array}\right\}
$$

where $F(Z, \theta)$ is a highly nonlinear function. The velocity field $\left(v_{x}, v_{y}\right)$ is given by a single vortex centered on the planar interface between the two media, which varies strongly in time and space.

The physics of the phenomenon can be briefly described as follows. A rotating vortex is introduced immediately at $t=0$. The resulting forced convection superposes to the diffusive mechanisms and accelerates the mixture of the gases. As a consequence, a diffusion flame ignites along the contact surface of both media, taking into account the important difference of temperatures in those regions. If the velocity field is sufficiently strong, it will entrain fresh gases into the vortex core which will react with an intensity set by the mixing temperature of gases of about $\left(T_{\mathrm{F}, 0}+T_{\mathrm{O}, 0}\right) / 2$. These locally lower temperatures result in a delayed ignition of the core unless air of sufficiently high temperature is initially considered. Once the flame is completely ignited, it propagates outwards from the center of the computational domain. The complete phenomenon encompasses thus very different physical regimes like mixing, ignition, propagation, which can be characterized depending on the initial reactants configuration and on the imposed velocity field, as studied in detail in [53].

As an illustration we recall a configuration investigated in [26] with fresh fuel initially at $T_{\mathrm{F}, 0}$ equal to $300 \mathrm{~K}$ and hot air at a temperature $T_{\mathrm{O}, 0}$ of $1000 \mathrm{~K}$, with a strongly varying velocity field. Figure 1 shows three different stages of the ignition phenomenon in terms of the heat release rate $F(Z, \theta)$. The adaptive splitting scheme of $\S 2$ with a predefined accuracy tolerance of $\eta=10^{-3}$, yields splitting time steps as shown in Figure 1. An initial splitting time step of $\Delta t=10^{-8}$ is chosen to properly handle the inclusion of the vortex and the fast variation of the velocity field. The splitting step increases until $t \approx 6.5 \times 10^{-5}\left(\Delta t \approx 2 \times 10^{-5}\right)$ during the mixing phase, and one then finds a series of rejected steps. The splitting time step is thus reduced down to the time scale needed to guarantee the prescribed accuracy: $\Delta t \approx 10^{-7}$. This behavior naturally coincides with the sudden ignition of the flame and the subsequent fast propagation along the contact surface, once a certain temperature is locally reached after the initial mixing of reactants. The last part shown in Figure 1 corresponds to the beginning of the propagation stage with $\Delta t \approx 10^{-5}$, where the core has not ignited yet.

A dynamic adaptation of the splitting time step is hence mandatory to identify these changes in the physical behavior of the phenomenon and to suitably describe the entire process. In particular 

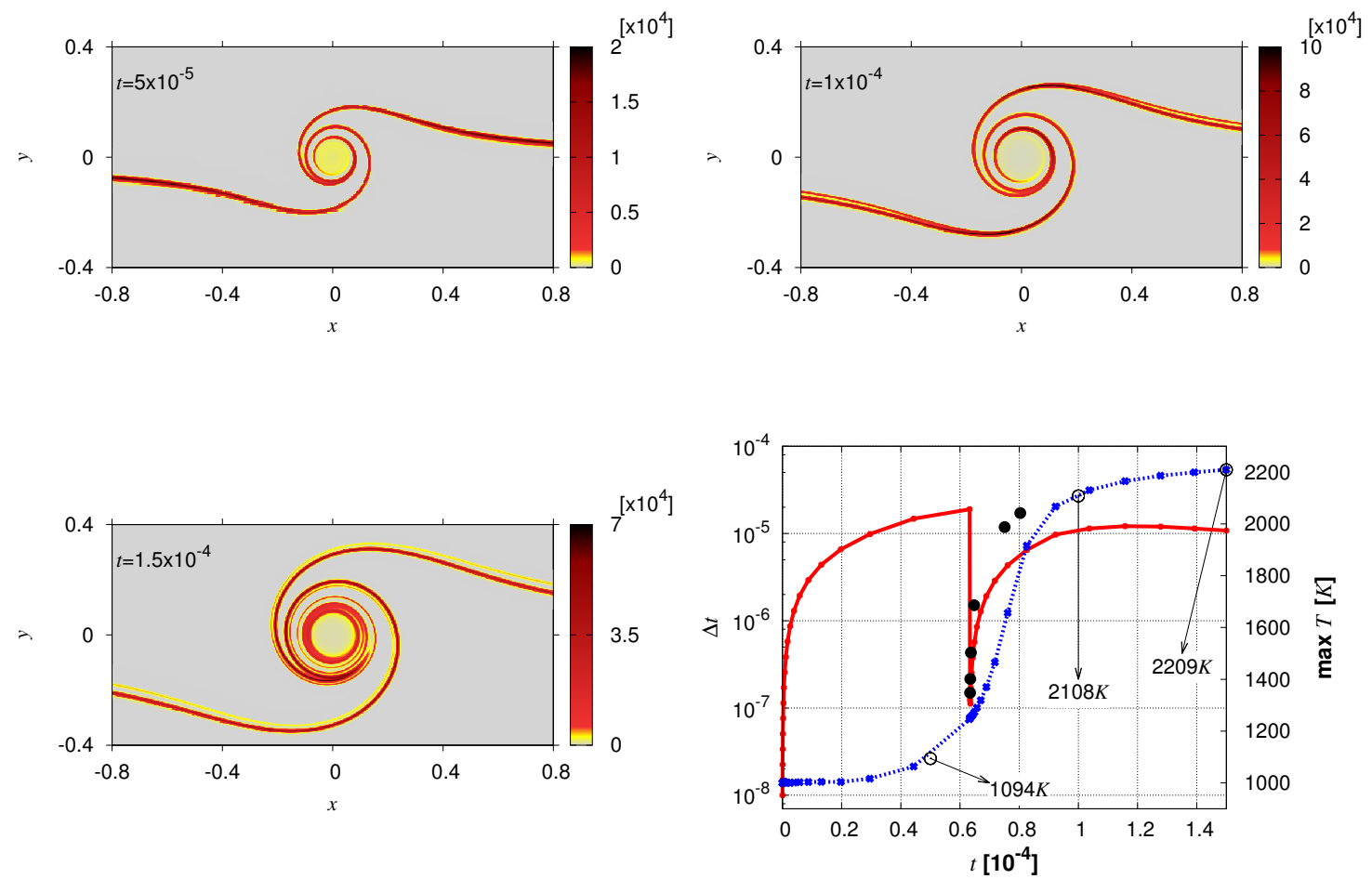

Figure 1: Instantaneous heat release rate $F$ at $t=5 \times 10^{-5}$ (top left), $10^{-4}$ (top right), and $1.5 \times 10^{-4}$ (bottom left). Bottom right: time evolution of splitting time steps and maximum temperature $T$, deduced from $\theta$. Rejected time steps are indicated with black bullets $(\bullet)$, while maximum temperatures for previous snapshots are marked with (o).

it takes approximately 207.5 minutes to solve this problem with an adaptive splitting technique on a uniform grid, compared to 674.7 minutes with a constant splitting time step of $10^{-7}$, of the order of the convective time steps. Greater gains are observed for longer periods of time integration as the impact of the initial transients and hence small splitting time steps is less important on the overall computational performance. Using dynamic grid adaptation combined with the adaptive splitting solver further reduces the CPU time to 8.9 minutes, taking into account the highly localized flame fronts in this particular problem. Most importantly, with this time-space adaptive technique time integration errors can be tracked and controlled as well as those originating from the compressed spatial representations.

\section{Conclusion}

In this chapter we have introduced operator splitting methods with error estimators and a time adaptive technique. The latter was further coupled with a space adaptive, finite volume multiresolution method. Numerical results obtained with this time-space adaptive technique support the conclusions that different multi-scale physical configurations can be successfully simulated and that numerical errors can be effectively controlled. The time and space adaptive techniques are clearly independent allowing the compatibility with any space discretization scheme such as finite volumes, finite elements or discontinuous Galerkin methods. The method allows us also to exploit the current 
computational resources and to obtain high efficiency in terms of load balancing on parallel architectures as it is shown in [18], where a task-based parallelism is used on multi-core architectures in conjunction with a work stealing approach.

\section{References}

[1] A. Abdulle. Fourth order Chebyshev methods with recurrence relation. SIAM J. Sci. Comput., 23(6):2041-2054 (electronic), 2002.

[2] Winfried Auzinger, Othmar Koch, and Mechthild Thalhammer. Defect-based local error estimators for splitting methods, with application to Schrödinger equations, Part I: The linear case. J. Comput. Appl. Math., 236(10):2643-2659, 2012.

[3] J. Bell, M.J. Berger, J. Saltzman, and M. Welcome. Three-dimensional adaptive mesh refinement for hyperbolic conservation laws. SIAM J. Sci. Comput., 15:127-138, 1994.

[4] J.B. Bell, M.S. Day, A. Almgren, M. Lijewski, C. Rendleman, R. Cheng, and I. Shepherd. Simulation of lean premixed turbulent combustion. J. Phys. Conf. Ser., 46:1-15, 2006.

[5] J.B. Bell, M.S. Day, and J.F. Grcar. Numerical simulation of premixed turbulent methane combustion. Proc. Combust. Inst., 29(2):1987-1993, 2002.

[6] J.B. Bell, M.S. Day, J.F. Grcar, M.J. Lijewski, J.F. Driscoll, and S.A. Filatyev. Numerical simulation of a laboratory-scale turbulent slot flame. Proc. Combust. Inst., 31(1):1299-1307, 2007.

[7] J.B. Bell, M.S. Day, I.G. Shepherd, M.R. Johnson, R.K. Cheng, J.F. Grcar, V.E. Beckner, and M.J. Lijewski. Numerical simulation of a laboratory-scale turbulent V-flame. Proc. Nat. Acad. Sci., 1021:10006-10011, 2005.

[8] M.J. Berger and J. Oliger. Adaptive mesh refinement for hyperbolic partial differential equations. J. Comput. Phys., 53:484-512, 1984.

[9] S. Blanes, F. Casas, P. Chartier, and A. Murua. Optimized high-order splitting methods for some classes of parabolic equations. Math. Comp., 82(283):1559-1576, 2013.

[10] Peter N. Brown, G.D. Byrne, and A.C. Hindmarsh. VODE: A variable-coefficient ODE solver. SIAM J. Sci. Stat. Comput., 10:1038-1051, 1989.

[11] F. Castella, P. Chartier, S. Descombes, and G. Vilmart. Splitting methods with complex times for parabolic equations. BIT Numer. Math., 49:487-508, 2009.

[12] A. Cohen, S.M. Kaber, S. Müller, and M. Postel. Fully adaptive multiresolution finite volume schemes for conservation laws. Math. Comp., 72:183-225, 2003.

[13] Alberto Cuoci, Alessio Frassoldati, Tiziano Faravelli, and Eliseo Ranzi. Numerical modeling of laminar flames with detailed kinetics based on the operator-splitting method. Energy $\& 3$ Fuels, 27(12):7730-7753, 2013.

[14] G. Dahlquist. A special stability problem for linear multistep methods. Nordisk Tidskr. Informations-Behandling, 3:27-43, 1963.

[15] Y. D'Angelo and B. Larrouturou. Comparison and analysis of some numerical schemes for stiff complex chemistry problems. RAIRO Modél. Math. Anal. Numér., 29(3):259-301, 1995.

[16] M.S. Day and J.B. Bell. Numerical simulation of laminar reacting flows with complex chemistry. Combust. Theory Model., 4:535-556, 2000. 
[17] S. Descombes. Convergence of a splitting method of high order for reaction-diffusion systems. Math. Comp., 70(236):1481-1501, 2001.

[18] S. Descombes, M. Duarte, T. Dumont, T. Guillet, V. Louvet, and M. Massot. Task-based adaptive multiresolution for time-space multi-scale reaction-diffusion systems on multi-core architectures. arXiv preprint arXiv:1506.04651, page 24, 2015.

[19] S. Descombes, M. Duarte, T. Dumont, V. Louvet, and M. Massot. Adaptive time splitting method for multi-scale evolutionary partial differential equations. Confluentes Math., 3(3):413443, 2011.

[20] S. Descombes and M. Massot. Operator splitting for nonlinear reaction-diffusion systems with an entropic structure: Singular perturbation and order reduction. Numer. Math., 97(4):667$698,2004$.

[21] Stéphane Descombes, Max Duarte, Thierry Dumont, Frédérique Laurent, Violaine Louvet, and Marc Massot. Analysis of operator splitting in the nonasymptotic regime for nonlinear reaction-diffusion equations. Application to the dynamics of premixed flames. SIAM J. Numer. Anal., 52(3):1311-1334, 2014.

[22] Stéphane Descombes and Michelle Schatzman. Strang's formula for holomorphic semi-groups. J. Math. Pures Appl. (9), 81(1):93-114, 2002.

[23] Stéphane Descombes and Mechthild Thalhammer. The Lie-Trotter splitting for nonlinear evolutionary problems with critical parameters: A compact local error representation and application to nonlinear Schrödinger equations in the semiclassical regime. IMA J. Numer. Anal., 33(2):722-745, 2013.

[24] Boun Oumar Dia and Michelle Schatzman. Commutateurs de certains semi-groupes holomorphes et applications aux directions alternées. RAIRO Modél. Math. Anal. Numér., 30(3):343383,1996 .

[25] M. Duarte. Méthodes numériques adaptatives pour la simulation de la dynamique de fronts de réaction multi-échelles en temps et en espace. PhD thesis, Ecole Centrale Paris, France, 2011.

[26] M. Duarte, S. Descombes, C. Tenaud, S. Candel, and M. Massot. Time-space adaptive numerical methods for the simulation of combustion fronts. Combust. Flame, 160:1083-1101, 2013.

[27] M. Duarte, M. Massot, S. Descombes, C. Tenaud, T. Dumont, V. Louvet, and F. Laurent. New resolution strategy for multi-scale reaction waves using time operator splitting and space adaptive multiresolution: Application to human ischemic stroke. ESAIM: Proc., 34:277-290, 2011.

[28] M. Duarte, M. Massot, S. Descombes, C. Tenaud, T. Dumont, V. Louvet, and F. Laurent. New resolution strategy for multiscale reaction waves using time operator splitting, space adaptive multiresolution and dedicated high order implicit/explicit time integrators. SIAM J. Sci. Comput., 34(1):A76-A104, 2012.

[29] T. Dumont, M. Duarte, S. Descombes, M.-A. Dronne, M. Massot, and V. Louvet. Simulation of human ischemic stroke in realistic 3D geometry. Commun. Nonlinear Sci. Numer. Simul., 18(6):1539-1557, 2013.

[30] G. Goyal, P.J. Paul, H.S. Mukunda, and S.M. Deshpande. Time dependent operator-split and unsplit schemes for one dimensional premixed flames. Combust. Sci. Technol., 60:167-189, 1988. 
[31] E. Hairer, C. Lubich, and G. Wanner. Geometric Numerical Integration. Structure-Preserving Algorithms for Ordinary Differential Equations. Springer-Verlag, Berlin, 2nd edition, 2006.

[32] E. Hairer and G. Wanner. Solving Ordinary Differential Equations II. Stiff and DifferentialAlgebraic Problems. Springer-Verlag, Berlin, 2nd edition, 1996.

[33] E. Hansen and A. Ostermann. High order splitting methods for analytic semigroups exist. BIT Numer. Math., 49:527-542, 2009.

[34] A. Harten. Multiresolution algorithms for the numerical solution of hyperbolic conservation laws. Comm. Pure Appl. Math., 48:1305-1342, 1995.

[35] W. Hundsdorfer and J.G. Verwer. Numerical Solution of Time-Dependent Advection-DiffusionReaction Equations. Springer-Verlag, Berlin, 2003.

[36] O.M. Knio, H.N. Najm, and P.S. Wyckoff. A semi-implicit numerical scheme for reacting flow. II. Stiff, operator-split formulation. J. Comput. Phys., 154(2):428-467, 1999.

[37] O. Koch, Ch. Neuhauser, and M. Thalhammer. Embedded exponential operator splitting methods for the time integration of nonlinear evolution equations. Appl. Numer. Math., 63:14$24,2013$.

[38] R. Kozlov, A. Kværnø, and B. Owren. The behaviour of the local error in splitting methods applied to stiff problems. J. Comput. Phys., 195(2):576-593, 2004.

[39] D. Lanser and J.G. Verwer. Analysis of operator splitting for advection-diffusion-reaction problems from air pollution modelling. J. Comput. Appl. Math., 111(1-2):201-216, 1999.

[40] G.I. Marchuk. Some application of splitting-up methods to the solution of mathematical physics problems. Appl. Math., 13(2):103-132, 1968.

[41] S. Müller. Adaptive Multiscale Schemes for Conservation Laws, volume 27 of Lect. Notes Comput. Sci. Eng. Springer-Verlag, 2003.

[42] H.N. Najm and O.M. Knio. Modeling low Mach number reacting flow with detailed chemistry and transport. J. Sci. Comput., 25(1-2):263-287, 2005.

[43] E.S. Oran and J.P. Boris. Numerical Simulation of Reacting Flows. Cambridge University Press, 2nd edition, 2001.

[44] Zhuyin Ren, Chao Xu, Tianfeng Lu, and Michael A. Singer. Dynamic adaptive chemistry with operator splitting schemes for reactive flow simulations. J. Comput. Phys., 263(0):19-36, 2014.

[45] D.L. Ropp and J.N. Shadid. Stability of operator splitting methods for systems with indefinite operators: Reaction-diffusion systems. J. Comput. Phys., 203(2):449-466, 2005.

[46] C. Safta, J. Ray, and H.N. Najm. A high-order low-Mach number AMR construction for chemically reacting flows. J. Comput. Phys., 229(24):9299-9322, 2010.

[47] D.A. Schwer, P. Lu, W.H. Green, and V. Semião. A consistent-splitting approach to computing stiff steady-state reacting flows with adaptive chemistry. Combust. Theory Model., 7(2):383399, 2003.

[48] Qin Sheng. Global error estimates for exponential splitting. IMA J. Numer. Anal., 14(1):27-56, 1994.

[49] M.A. Singer and S.B. Pope. Exploiting ISAT to solve the reaction-diffusion equation. Combust. Theory Model., 8(2):361-383, 2004. 
[50] M.A. Singer, S.B. Pope, and H.N. Najm. Modeling unsteady reacting flow with operator splitting and ISAT. Combust. Flame, 147(1-2):150-162, 2006.

[51] B. Sportisse. An analysis of operator splitting techniques in the stiff case. J. Comput. Phys., 161(1):140-168, 2000.

[52] G. Strang. On the construction and comparison of difference schemes. SIAM J. Numer. Anal., 5:506-517, 1968.

[53] D. Thévenin and S. Candel. Ignition dynamics of a diffusion flame rolled up in a vortex. Phys. Fluids, 7(2):434-445, 1995.

[54] H.F. Trotter. On the product of semi-groups of operators. Proc. Am. Math. Soc., 10:545-551, 1959.

[55] M. Valorani and D.A. Goussis. Explicit time-scale splitting algorithm for stiff problems: Autoignition of gaseous mixtures behind a steady shock. J. Comput. Phys., 169(1):44-79, 2001.

[56] P.J. van der Houwen and B.P. Sommeijer. On the internal stability of explicit, $m$-stage RungeKutta methods for large m-values. Z. Angew. Math. Mech., 60(10):479-485, 1980.

[57] J. G. Verwer, E. J. Spee, J. G. Blom, and W. Hundsdorfer. A second-order Rosenbrock method applied to photochemical dispersion problems. SIAM J. Sci. Comput., 20(4):1456-1480, 1999.

[58] J.G. Verwer. Explicit Runge-Kutta methods for parabolic partial differential equations. Appl. Numer. Math., 22(1-3):359-379, 1996.

[59] B. Yang and S.B. Pope. An investigation of the accuracy of manifold methods and splitting schemes in the computational implementation of combustion chemistry. Combust. Flame, 112(1-2):16-32, 1998. 